\title{
Structural assessment of a whole toroidal sector of the HELIAS 5-B breeding blanket
}

Gaetano Bongioví $^{1}$, André Häußler ${ }^{1}$, Salvatore Giambrone ${ }^{2}$, Ilenia Catanzaro², Ruggero Forte $^{2}$, Guangming Zhou ${ }^{1}$, Pietro Alessandro Di Maio ${ }^{2}$ and the W7-X team

${ }^{1}$ Karlsruhe Institute of Technology, INR, Germany - 2Universitá degli Studi di Palermo, Dipartimento di Ingegneria, Italy

The European roadmap for the realization of fusion energy considers the stellarator line as a possible long-term alternative to a tokamak DEMO. Presently, the most promising option is a five-field period power plant called HELIcal-axis Advanced Stellarator (HELIAS) 5-B. It is a direct extrapolation of the Wendelstein 7-X, the largest stellarator reactor currently in operation. In order to allow the electricity production, the HELIAS 5-B reactor must be endowed with a breeding blanket (BB). Hence, in this paper, the advancements in the HELIAS 5-B BB design are reported.
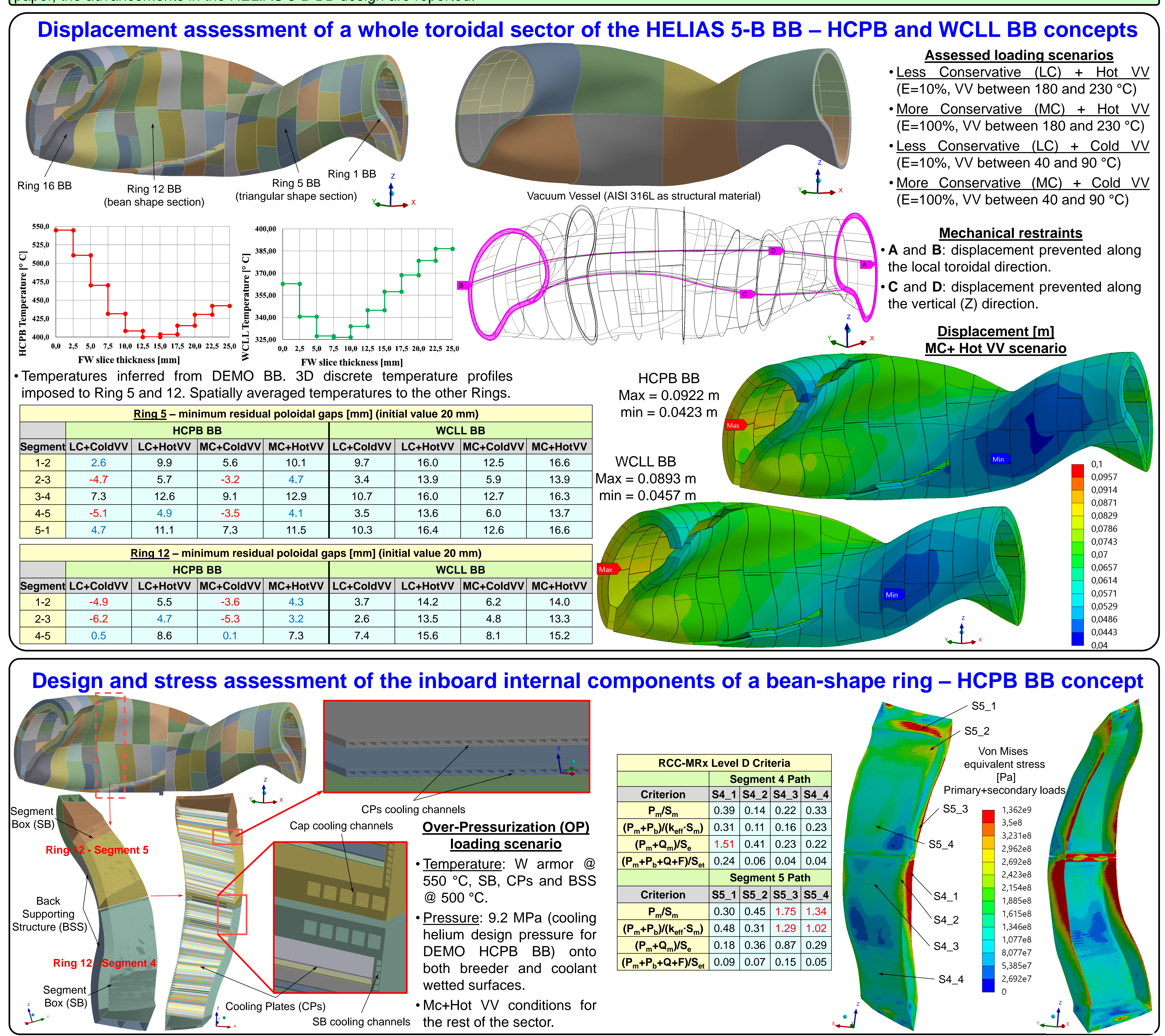

Firstly, a displacement assessment of a whole toroidal sector of the HELIAS 5-B BB has been performed considering both WCLL and HCPB BB concepts. Homogenized geometric models have been set-up, adopting dummy components (full blocks without internal details). Results allow concluding that the proposed BB segmentation strategy is viable for more detailed BB design. Then, focusing on the HCPB BB concept, a preliminary design of internal components in the inboard blanket segments of the Ring 12 has been carried out. Structural analysis under the reference over-pressurization accidental conditions have been performed. Comparing the calculated stress against the RCC-MRx structural design criteria, one allows concluding that the adopted design approach is promising. Nevertheless, a more detailed assessment is necessary to develop design modifications aimed at improving the blanket structural performances, especially in the most critical regions highlighted by the present work. 